\title{
ARTIGO CIENTÍFICO \\ Dinâmica do fitoplâncton e evolução trófica de reservatório no Semiárido brasileiro
}

\section{Fitoplâncton dynamics and tropical reservoir evolution in brazilian Semi-arid}

\author{
Patrícia Silva Cruz ${ }^{1 *}$, Ranielle Daiana Dos Santos Silva ${ }^{2}$, Dayany Aguiar de Oliveira ${ }^{3}$, Leandro Gomes Viana ${ }^{4}$, Daniely de \\ Lucena Silva ${ }^{5}$, José Etham de Lucena Barbosa ${ }^{6}$
}

\begin{abstract}
Resumo: O presente estudo objetivou analisar a dinâmica de grupos funcionais do fitoplâncton e a variação do grau de trofia em reservatório do semiárido brasileiro durante estiagem prolongada. O estudo foi realizado no reservatório de Cordeiro, no município do Congo, Paraíba, com amostragens no período de junho a dezembro de 2015, com frequência trimestral, no ponto próximo ao barramento do reservatório. As espécies do fitoplâncton foram identificadas e quantificadas para obtenção da densidade, posteriormente as espécies foram classificadas de acordo com suas estratégias e grupos funcionais. $\mathrm{O}$ estado trófico foi determinado à partir do Índice do estado trófico para ambientes tropicais. O período de estudo foi caracterizado pela redução dos níveis de água acumulados no reservatório. A transparência do Secchi foi baixa, cujos valores foram inferiores a $1 \mathrm{~m}$. As concentrações de fósforo total durante o período de estudo foram elevadas (acima de $200 \mu \mathrm{g} / \mathrm{L}$ ). As concentrações de clorofila $a$, tiveram valores mínimos de $8,17 \mu \mathrm{g} / \mathrm{L}$ em julho de 2015 , e máximos de $93,67 \mu \mathrm{g} / \mathrm{L}$ em outubro de 2015 , com oscilações durante todo o período de estudo. $\mathrm{O}$ ambiente foi classificado como mesotrófico no mês de junho e eutrófico nos meses de outubro e dezembro. Foram identificadas dez espécies descritoras, agrupadas em quatro estratégias de vida (CS, C, R, e S) e 08 grupos funcionais (D, F, H1, N, P, X1 e Z). Os grupos funcionais refletiram as mudanças ambientais, sendo notória a presença de grupos típicos de lagos rasos meso-eutróficos. As espécies potencialmente tóxicas encontradas no estudo podem representar riscos à saúde das pessoas que utilizam essa água para consumo.
\end{abstract}

Palavras-chave: Eutrofização; Cianobactérias; Lago Raso.

Abstract: The present study aimed to analyze the dynamics of phytoplankton functional groups and the degree of trophic degree in the Brazilian semi - arid reservoir during prolonged drought. The study was carried out in the Cordeiro reservoir, in the municipality of Congo, Paraiba, with samples from June to December 2015, with a quarterly frequency, near the reservoir bus. The phytoplankton species were identified and quantified to obtain the density, later the species were classified according to their strategies and functional groups. The trophic state was determined from the Index of the trophic state, for tropical environments. The study period was characterized by the reduction of water levels accumulated in the reservoir. The transparency of the Secchi was low, whose values were less than $1 \mathrm{~m}$. Total phosphorus concentrations during the study period were high (above $200 \mu \mathrm{g} / \mathrm{L}$ ). Chlorophyll-a concentrations had a minimum of $8.17 \mu \mathrm{g} / \mathrm{L}$ in July 2015 and a peak of 93.67 $\mu \mathrm{g} / \mathrm{L}$ in October 2015, with fluctuations throughout the study period. The environment was classified as mesotrophic in the month of June and eutrophic in the months of October and December. Ten descriptive species, grouped into four life strategies $(\mathrm{CS}, \mathrm{C}, \mathrm{R}$, and $\mathrm{S})$ and 08 functional groups $(\mathrm{D}, \mathrm{F}, \mathrm{H} 1, \mathrm{~N}, \mathrm{P}, \mathrm{X} 1$ and $\mathrm{Z})$ were identified. The functional groups reflected the environmental changes, being typical the presence of typical groups of meso-eutrophic shallow lakes. The potentially toxic species found in the study may pose health risks to people who use this water for consumption.

Keywords: Eutrophication; Cyanobacteria; Shallow Lake.

\footnotetext{
*Autor para correspondência

Recebido para publicação em 07/02/2018; aprovado em 17/09/2018

${ }^{1}$ Biologa. Doutoranda em Engenharia Ambiental pela Universidade Estadual da Paraíba,. Campina Grande. E-mail:patriciacruz_biologa@hotmail.com

${ }^{2}$ Biologa, Mestranda em Ecologia pela Universidade Estadual da Paraíba, E-mail: ranielledaiana@ hotmail.com.

${ }^{3}$ Biologa, Mestranda em Ecologia pela Universidade Estadual da Paraíba, E-mail:dayanyaguiar93@gmail.com.

${ }^{4}$ Biólogo, Mestre em Ciência e Tecnologia Ambiental pela Universidade Estadual da Paraíba, E-mail:leandrogomesbiologo@gmail.com.

${ }^{5}$ Química, Mestre em Ciência e Tecnologia Ambiental pela Universidade Estadual da Paraíba, E-mail: danyquimicg@gmail.com

${ }^{6}$ Biológo. Doutor em Ecologia, Universidade Estadual da Paraíba; E-mail: ethambarbosa@ hotmail.com
} 


\section{INTRODUÇÃO}

A região Nordeste do Brasil é caracterizada por grande escassez hídrica, altas temperaturas e precipitações irregulares (COSTA et al., 2016). Para suprir a necessidade hídrica durante o período de seca, é comum a construção de reservatórios. Estes reservatórios são destinados a usos múltiplos, dentre eles estão dessedentação animal, irrigação, pescas interiores e o consumo humano. Esses ambientes são fortemente influenciados pela baixa velocidade de ventos, grandes perdas por evaporação e alto tempo de residência da água (CHELLAPPA et al., 2009), tendo como consequências a redução do volume d'água e o aumento das concentrações de nutrientes (NASELLI-FLORES et al., 2007).

Uma revisão recente de estudos realizados em lagos e reservatórios rasos (BARBOSA; FRANÇA, 2011; BONILA et al., 2012; COSTA et al., 2016) indicou que a redução do nível de água muitas vezes resulta em concentrações mais elevadas de nutrientes, maior biomassa do fitoplâncton e redução da transparência da água (JEPPESEN et al., 2015).

Altas concentrações de nutrientes, sobretudo fosfatados e nitrogenados, e baixa transparência da água, aliadas à alta taxa de irradiação e às elevadas temperaturas, favorecem o intenso crescimento de cianobactérias durante todo o ano em mananciais utilizados para abastecimento humano (ROMO et al., 2013).

Dentre os problemas de maior impacto atual na saúde pública relacionado com a poluição dos corpos hídricos destaca-se a ocorrência cada vez mais frequente de florações algais com predominância de cianobactérias, em particular de espécies potencialmente produtora de cianotoxinas. Essa problemática encontra-se disseminada em corpos aquáticos por todo mundo, associada, principalmente, à disponibilidade de nutrientes nos mananciais (eutrofização), ao aumento da temperatura das superfícies da água como efeito das mudanças no clima mundial (O’NEIL et al., 2012).

Estudos sobre a dinâmica do fitoplâncton em regiões semiáridas têm demonstrado a persistência de florações de cianobactérias durante a maior parte do ano, em períodos secos (NASELLI-FLORES et al., 2007; DANTAS et al., 2011), em virtude de muitos gêneros apresentarem vantagens adaptativas para sobreviver em ambientes com altas temperaturas, intensidades luminosas e elevadas concentrações de nutrientes (PELECHATA et al., 2016).

A fim de se prever os efeitos das mudanças naturais ou antrópicas nos reservatórios, tem-se intensificado a busca por esquemas de classificação que possam ser usados para simplificar a complexidade do ecossistema. As tentativas de classificar as comunidades por suas características adaptativas e morfológicas são cada vez mais frequentes, onde as comunidades do fitoplâncton podem ser caracterizadas por sua composição de grupo taxonômico ou funcional (SALMASO et al., 2014).

A proposta de um esquema de classificação funcional de espécies do fitoplâncton por Reynolds (1997) e Reynolds et al. (2002) foi testada e aprovada como importante ferramenta de poder preditivo (MARINHO; HUSZAR, 2002; KRUK et al., 2002; FONSECA, 2005), que categoriza espécies com semelhanças morfológicas, fisiológicas e ecológicas em grupos funcionais nomeados a partir de caracteres alfanuméricos. Esse esquema é amplamente utilizado em todo mundo (BECKER et al., 2009) e permite classificar as espécies em grupos diretamente relacionados com $\mathrm{O}$ funcionamento do ecossistema, além de fornecer informações sobre a dinâmica das espécies. Diante o exposto, objetivou-se com esse estudo, analisar a dinâmica de grupos funcionais do fitoplâncton e a variação do grau de trofia em um reservatório do semiárido brasileiro em período caracterizado por estiagem prolongada.

\section{MATERIAL E MÉTODOS}

O estudo foi realizado no reservatório Cordeiro, localizado na região do alto Paraíba $\left(7^{\circ} 47^{\prime} 38.00^{\prime \prime} \mathrm{S}\right.$ 36 40'14.04" W), com capacidade de acumulação de $69.965 .945 \mathrm{~m}^{3}$ (Figura 1). Abastece os municípios do Congo (cidade onde está localizado), integrando um sistema adutor que distribui águas para o abastecimento das cidades de Monteiro, Sumé, Serra Branca e São João do Cariri. É utilizado para fins de abastecimento, irrigação, dessedentação de rebanhos, pesca e em algumas iniciativas de lazer e turismo regional.

Figura 1. Imagem do reservatório de Cordeiro, município do Congo-PB, durante período de estiagem prolongada.

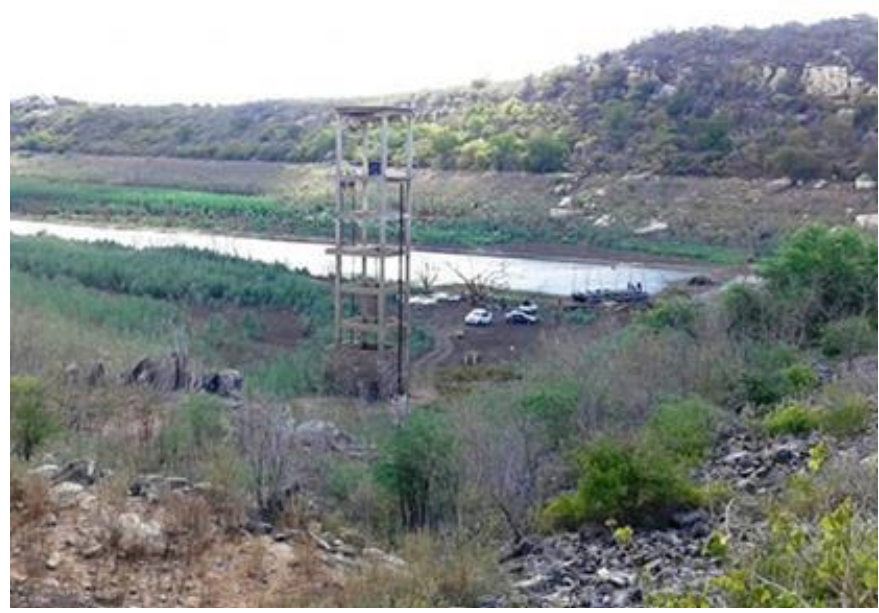

Foto: Patrícia Silva Cruz, 2015.

Os dados de volume do reservatório foram disponibilizados pela Agência Executiva de Gestão das Águas do Estado da Paraíba (AESA).

As amostragens foram realizadas com frequência trimestral durante o período de junho a dezembro de 2015, no ponto próximo ao barramento do reservatório. As amostras de água $(500 \mathrm{~mL})$ foram coletadas na sub-superfície $(100 \%$ de penetração da luz) e acondicionadas em garrafas plásticas e preservadas em gelo (nutrientes) e com Lugol (fitoplâncton).

As análises foram realizadas no Laboratório de Ecologia Aquática - LEAq, situado na Universidade Estadual da Paraíba (UEPB).

Foram medidas através de sonda multiparamétrica (Horiba, U-50) as seguintes variáveis: temperatura e pH. A transparência da água, foi medida através da profundidade de extinção obtida com o disco de Secchi. Para determinação da concentração de clorofila $a$ as amostras de água foram filtradas em filtros de fibra de vidro (GF/C Whatman) e posteriormente extraídas com etanol a $96 \%$ e medidas por espectrofotometria (JESPERSEN; CHRISTOFFERSEN, 1987).

As análises para nutrientes dissolvidos inorgânicos (fósforo solúvel reativo) foram inicialmente filtradas em 
filtros de fibra de vidro ( $\varnothing 47 \mathrm{~mm}$ e $1,5 \mu \mathrm{m}$ de porosidade) e determinado pelo método de oxirredução por persulfato de potássio com leitura espectrofotométrica. Para determinação do fósforo total, utilizou-se o método de oxirredução por persulfato de potássio e leitura espectrofotométrica. Todas as análises seguiram as recomendações descritas no Standard Methods for the Examination of Water and Wastewater (APHA, 2012).

O Índice de Estado Trófico (IET) foi determinado seguindo o índice de Carlson (1977), modificado para ambientes tropicais por Toledo Júnior et al. (1983). A classificação trófica segue o critério: oligotrófico quando o IET < 44; mesotrófico com IET 54 < IET > 44 e eutrófico quando o IET $>54$.

As análises quantitativas seguiram o método de sedimentação de Uthermöhl (1958). O sistema de classificação para gêneros e classes seguiram as recomendações de Bicudo e Menezes (2006), e para a identificação das espécies utilizaram-se chaves de identificações de cada grupo. A densidade foi estimada pela fórmula descrita por Ross (1979).

O biovolume de algas $\left(\mathrm{mm}^{3} \mathrm{~L}^{-1}\right)$ foi calculado utilizando formas (HILLEBRAND et al., 1999). Foram medidos de 20 a 30 espécimes ou colônias em média de cada táxon, expressos em unidade de peso fresco em massa, $1 \mathrm{~mm}^{3} 1-1=1 \mathrm{mg} \cdot \mathrm{L}^{-1}$ (WETZEL; LIKENS, 2000). As espécies descritoras foram definidas como aquelas cuja contribuição foi de $5 \%$ da biomassa total de algas e então classificadas em grupos funcionais usando os critérios de Reynolds et al. (2002) e Padisák et al. (2009).

\section{RESULTADOS E DISCUSSÃO}

Com base nos dados dos últimos 10 (dez) anos (Figura 2), pode-se observar que o período de estudo foi caracterizado pela redução dos níveis de água acumulada no reservatório Cordeiro. Durante a estação seca, considerada para a região, a frequência e a intensidade de precipitação permaneceram baixas. A irregularidade e/ou deficiência das chuvas, assim como a elevada taxa de evapotranspiração são características das regiões semiáridas e provocam a perda de grande parte das águas superficiais da região (SILVA, 2007), fato que contribui para as condições mais eutróficas de corpos aquáticos dessa região.

A temperatura da água durante o período de observação apresentou valores médios de $23^{\circ} \mathrm{C}$. O comportamento observado pode ser decorrente da baixa profundidade do ambiente e ação dos ventos que ocasionam o resfriamento pela perda de calor da superfície da água para a atmosfera.

O pH básico observado durante o estudo $(7,57$ e 9,87), pode ser justificado pelo intenso processo de mistura da coluna d'água e pela influência da composição química da bacia de drenagem do reservatório (LEITÃO et al., 2006).

A transparência da água foi baixa, com valores inferiores a $1 \mathrm{~m}$. A permanência da baixa transparência da água funcionou como fator relevante para o favorecimento de cianobactérias filamentosas como a Cylindrospermopsis raciborskii, que possui várias habilidades, dentre elas, o baixo requerimento de luz (BRIAND et al., 2004; BUFORD; DAVIS, 2011).

A concentração de fósforo total durante o período de estudo foi elevada (acima de $200 \mu \mathrm{g} / \mathrm{L}$ ). A concentração de clorofila $a$, apresentou valores mínimos de $8,17 \mu \mathrm{g} / \mathrm{L}$ em julho de 2015, e máximos de 93,67 $\mu \mathrm{g} / \mathrm{L}$ em outubro de 2015, com oscilações durante todo o período de estudo. A concentração do fósforo reativo solúvel variou de $9,31 \mu \mathrm{g} / \mathrm{L}$ em julho de 2015 a 76,45 $\mu \mathrm{g} / \mathrm{L}$ em dezembro de 2015

Figura 2. Evolução do Volume Armazenado em $\mathrm{m}^{3}$ do Reservatório de Cordeiro no período de 2008 a 2017.

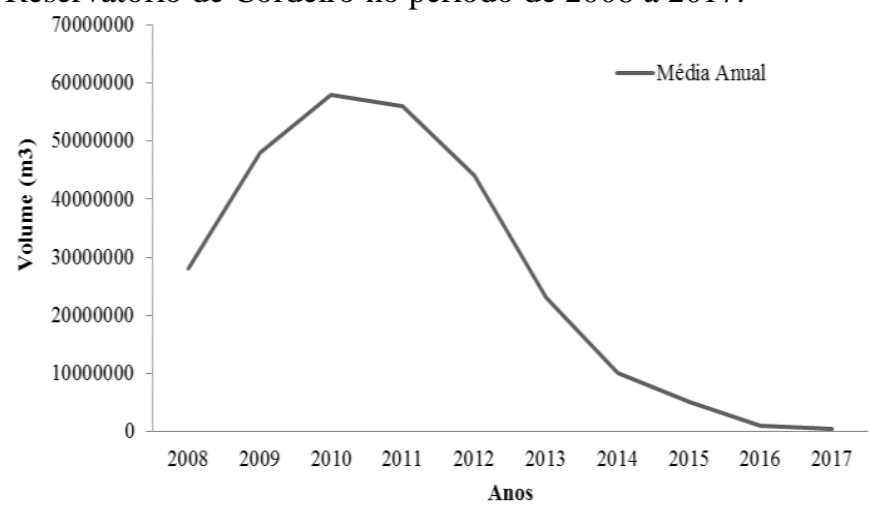

Estudos no semiárido brasileiro descrevem o aumento da biomassa e o predomínio de cianobactérias associados às condições mais rasas, eutróficas e túrbidas dos reservatórios (DANTAS et al. 2011; SOARES et al. 2013), demonstrando que luz e nutrientes são fatores cruciais para a produtividade e a dinâmica do fitoplâncton nos ambientes.

O grau de trofia do reservatório durante o período de estudo variou de mesotrófico, em julho/15 (Figura 3), para eutrófico nos meses de outubro/15 e dezembro/15. Segundo estudos realizados por Costa et al., (2009) e Medeiros et al. (2015), os sistemas em períodos caracterizados por estiagem prolongada, estão propensos a terem seu estado trófico aumentado para condições eutróficas. Esses autores sugerem que esse fato é decorrente da alta taxa de evaporação e concentração de nutrientes.

Figura 3. Classificação do Índice de Estado Trófico (IET) médio do reservatório de Cordeiro durante o período de julho a dezembro de 2015 .

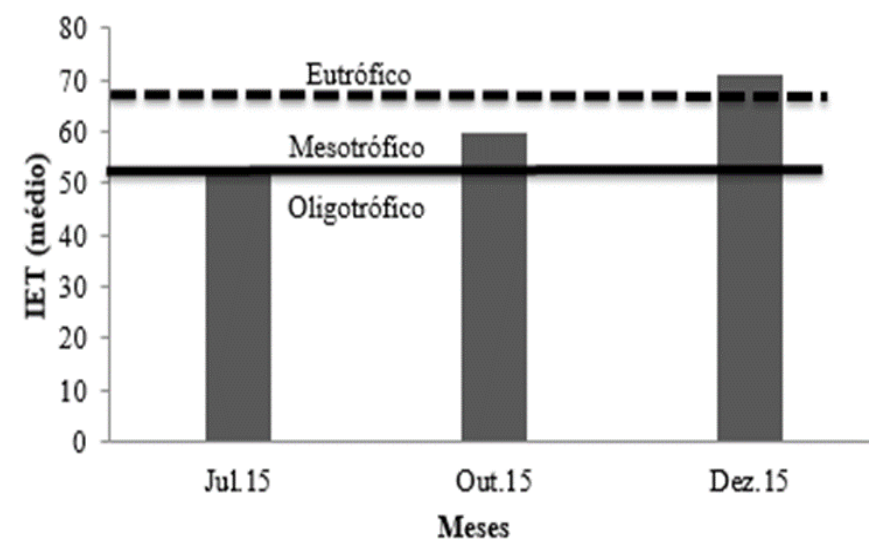

Quando analisada a composição da comunidade fitoplanctônica, foram identificados 26 táxons (Tabela 2), pertencentes às classes: Cyanobacteria (22\%), Chlorophyceae (34\%), Bacillariophyceae (4\%), Euglenophyceae (15\%) e Zygnemaphyceae (25\%). Dentre as espécies de cianobactérias, as mais abundantes foram: Aphanocaspa sp, Dolichospermum solitarium, Microcystis sp. e Planktothrix isothrix, consideradas espécies com potencial toxigênico. 
Figura 4. Densidade (ind $/ \mathrm{mL}$ ) das classes do fitoplâncton durante o período de julho a dezembro de 2015.

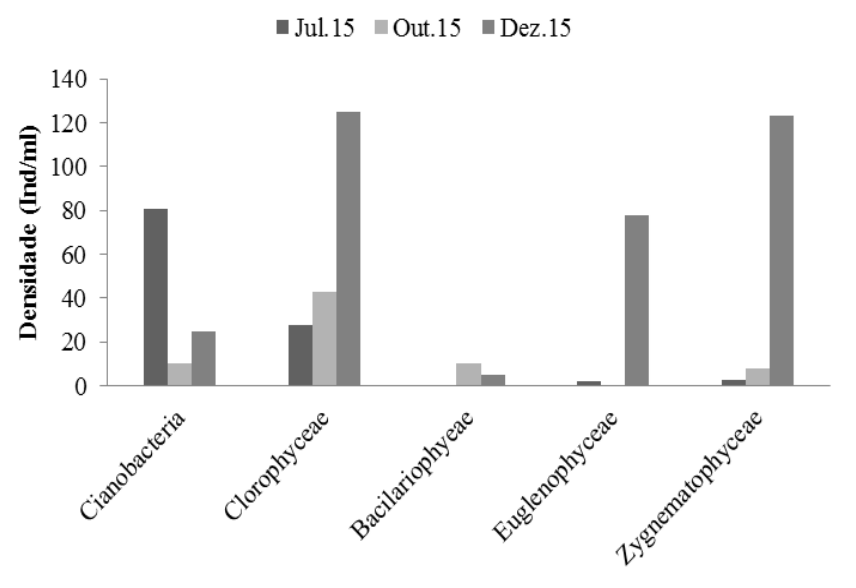

A espécie Planktothrix isothrix apresenta distribuição cosmopolita em águas eutróficas a hipereutróficas (KOMÁREK; KOMÁRKOVÁ-LEGNEROVÁ, 2002; KOMÁREK; ANAGNOSTIDIS, 2005). A espécie Cylindrospermopsis raciborskii está entre as cianobactérias formadoras de florações mais comuns em ambientes de água doce, com distribuição principalmente pantropical, apresentando grande plasticidade fenotípica e é uma espécie potencialmente tóxica, com linhagens capazes de produzir saxitoxinas e neosaxitoxinas (SANT'ANNA et al., 2008). A presença desta espécie fixadora de nitrogênio é associada a maior plasticidade de adaptações às variáveis ambientais (BONILLA et al., 2012; BITTENCOURT-OLIVEIRA et al., 2010). Entre as vantagens apresentadas por esta espécie, destaca-se sua capacidade de tolerar elevadas temperaturas da água, baixas concentrações de nutrientes, baixa transparência da água e elevados valores de salinidade e $\mathrm{pH}$, conforme ressaltado em estudos de Briand et al. (2002) e Bonilla et al. (2012). Além disso, estudos de Buford et al. (2006) ressaltam o sucesso dessa espécie, relacionado a sua capacidade de armazenar fósforo e de utilizar condições de baixa luminosidade para sua sobrevivência.

A presença das Chlorophyceaes foram relacionadas com os períodos de maior disponibilidade de nutrientes, provenientes da ressuspensão do sedimento (GENTIL et al., 2008).

Segundo Becker et al. (2009) a contribuição das algas verdes coloniais com bainha mucilaginosa, principalmente durante a fase de estratificação térmica, assemelha-se ao comportamento das cianobactérias formadoras de colônias.

A alta expressividade da classe Euglenophyceae (Figura 4) pode ser associada à baixa transparência da água e elevadas concentrações de nutrientes fosfatados. De acordo com estudos de Gentil (2007) e Lachi; Sipaúba-Tavares (2008), a boa expressividade dessa classe é favorecida pelas elevadas concentrações de nitrogênio e fósforo. Pode-se observar ainda, a contribuição da Zignemaphyceae (Figura 4), cujas espécies são encontradas em ambientes com águas alcalinas e ricas em matéria orgânica. $\mathrm{O} \mathrm{pH}$ mais alcalino em dezembro/15, foi determinante para a alta densidade de espécimes dessa classe.

À partir dos táxons identificados foram verificadas 10 espécies descritoras (biomassa $\geq 5 \%$ ): Aphanocapsa sp.; Chroococcus turgidus; Dolichospermum solitarium;
Monoraphidium irregulare; Monoraphidium contortum; Oocystis marsoni; Cyclotella meneghiniana; Cosmarium pseudoconnatum; Closterium kuetzingii e Closterium sp., agrupadas em quatro estratégias de vida (CS, C, R e S) e oito grupos funcionais (D, F, K, H1, N, P, X1 e Z) (Tabela 1), típicos de ambientes rasos, túrbidos e em condições mesohipertróficos (REYNOLDS et al., 2002; PADISÁK et al., 2009).

Embora as espécies tenham sido agrupadas nas estratégias CS, C, R e S, houve dominância e/ou coexistência de espécies $\mathrm{C}$ e $\mathrm{S}$ estrategistas. $\mathrm{O}$ sucesso das espécies $\mathrm{C}$ estrategistas deve-se a sua preferência por ambientes com altas concentrações de nutrientes, sendo mais competitivas em ambientes eutróficos e estáveis. As espécies S estrategistas, estiveram relacionadas com o período de maior luminosidade (Secchi) e altas concentrações de nutrientes.

$\mathrm{O}$ grupo funcional $\mathrm{D}$ representado pela espécie Cyclotella meneghiniana, remete períodos de condições eutróficas. Já o grupo $\mathrm{F}$ esteve composto por algas verdes coloniais tolerantes à mistura (REYNOLDS et al. 2002).

Tabela 1. Estratégias e Grupos Funcionais das espécies descritoras identificadas durante o período de estudo.

\begin{tabular}{|c|c|c|c|}
\hline $\mathrm{E}$ & ES & GF & AMBIENTE \\
\hline Aphanocapsa sp & $\mathrm{R}$ & K & $\begin{array}{l}\text { Ambientes } \\
\text { misturados e ricos em } \\
\text { nutrientes }\end{array}$ \\
\hline $\begin{array}{l}\text { Chroococcus } \\
\text { turgidus }\end{array}$ & S & $\mathrm{Z}$ & $\begin{array}{l}\text { Ambientes com altas } \\
\text { concentrações de } \\
\text { nutrientes e misturados }\end{array}$ \\
\hline $\begin{array}{l}\text { Dolichospermum } \\
\text { solitarium }\end{array}$ & CS & H1 & $\begin{array}{l}\text { Ambientes com baixo } \\
\text { teor de Nitrogênio }\end{array}$ \\
\hline $\begin{array}{l}\text { Monoraphidium } \\
\text { irregulare }\end{array}$ & CS & $\mathrm{X} 1$ & $\begin{array}{l}\text { Ambientes rasos, } \\
\text { hipereutróficos }\end{array}$ \\
\hline $\begin{array}{l}\text { Monoraphidium } \\
\text { contortum }\end{array}$ & $\mathrm{C}$ & $\mathrm{X} 1$ & $\begin{array}{l}\text { Ambientes rasos, eu- } \\
\text { hipereutróficos }\end{array}$ \\
\hline Oocystis marsoni & $\mathrm{C}$ & $\mathrm{F}$ & $\begin{array}{l}\text { Ambientes meso- } \\
\text { eutróficos }\end{array}$ \\
\hline $\begin{array}{l}\text { Cyclotella } \\
\text { meneghiniana }\end{array}$ & $\mathrm{R}$ & D & $\begin{array}{l}\text { Ambientes rasos, } \\
\text { túrbidos e enriquecidos }\end{array}$ \\
\hline $\begin{array}{l}\text { Cosmarium } \\
\text { psedocomatum }\end{array}$ & $\mathrm{R}$ & $\mathrm{N}$ & Epilímnio mesotrófico \\
\hline $\begin{array}{l}\text { Closterium } \\
\text { kuetzingii }\end{array}$ & $\mathrm{R}$ & $\mathrm{P}$ & Epilímnio eutrófico \\
\hline Closterium sp & $\mathrm{R}$ & $P$ & Epilímnio eutrófico \\
\hline
\end{tabular}

*E= espécie; $\mathrm{ES}=$ estratégia de vida; $\mathrm{GF}$ = grupo funcional.

As espécies pertencentes ao grupo $\mathrm{K}$ apresentaram elevados biomassa, expressa em biovolume e foi representado por organismos com afinidade a coluna d'água rica em nutrientes, a exemplo das cianobactérias coloniais Aphanocapsa sp. Em contrapartida, nos períodos com altas concentrações de nutrientes e mistura da coluna d'água, houve florações de organismos pequenos a exemplo do (Chroococcus turgidus) pertencente ao grupo Z.

Destacaram-se ainda com significativas contribuições as espécies classificadas nos grupos funcionais $\mathrm{K}$ e $\mathrm{H} 1$ pertencentes à classe Cyanobacteria, que são típicos de ambientes rasos, com coluna d'água misturada e ricos em nutrientes (PADISAK et al., 2009).

Nos grupos X1 e F, ocorreram espécies da classe Clorophyceae, que são típicas de ambientes rasos e meso, euhipertróficos. 
A espécie Cyclotella meneghiniana, pertencente à Bacilariophyceae é típica de ambientes túrbidos, rasos e enriquecidos (Grupo D) e as espécies da classe Zygnematophyceae que pertecem aos grupos $\mathrm{N}$ e $\mathrm{P}$, que são típicas de ambientes com epilímnio meso-eutróficos.

\section{CONCLUSÕES}

As flutuações no nível d’água armazenado no reservatório favorece à mudanças nas características físicas, químicas e biológicas. Essa condição, aliada à alta irradiação, às elevadas temperaturas e ao estado eutrófico, favoreceram o intenso crescimento e persistência das cianobactérias.

A aplicação dos grupos funcionais mostrou-se uma ferramenta robusta no entendimento da dinâmica da comunidade fitoplanctônica, onde as espécies registradas refletiram na multiplicidade do fitoplâncton que apresentou-se bem adaptado às condições típicas de ambientes misturados, enriquecidos e limitados por luz.

As espécies potencialmente tóxicas observadas nesse estudo, podem oferecer riscos à saúde da população que utiliza essa água para consumo, principalmente, sem tratamento prévio.

\section{REFERÊNCIAS}

APHA, AMERICAM PUBLIC HEALTH ASSOCIATION. Standard methods for the examination of water and wastewater. 19 ed. Washington D.C.: APHA - AWWA WPCF, 2012.

BARBOSA, J. E. L.; FRANÇA, J. C. Educação Ambiental e a conservação da biodiversidade aquática do semiárido. In: ABÍliO, F. J. P (Org.). Educação Ambiental para o semiárido. João Pessoa-PB: Editora Universitária da UFPB, 2011. cap. 8, p.359-384.

BECKER, V.; HUSZAR, V. L. M.; CROSSETTI, L. O. Responses of phytoplankton functional groups to the mixing regime in a deep subtropical reservoir. Hydrobiologia, v. 628, n. 1, p.137-15, 2009.

BICUDO, C. E. de M.; MENEZES, M. Gêneros de Algas de Águas Continentais do Brasil. 2.ed. São Carlos: Editora Rima, 2006. 489p.

BITTENCOURT-OLIVEIRA, M. C.; SANTOS, D. M. S.; MOURA, N. A. Toxic cyanobacteria in reservoirs in northeastern Brazil: detection using a molecular method. Brazilian Journal of Biology, v.70, n.4, p.1005-1010, 2010.

BONILLA, S.; AUBRIOT, L.; SOARES, M. C. S.; GONZÁLEZ-PIANA, M.; FABRE, A.; HUSZAR, V. L. M.; LÜRLING, M.; ANTONIADES, D.; PADISÁK, J.; KRUK, C. What drives the distribution of the bloom-forming cyanobacteria Planktothrix agardhii and Cylindrospermopsis raciborskii? FEMS Microbiology Ecology, v.79, n.3, p.594607, 2012.

BRIAND, J. F.; ROBILLOT, C.; QUIBLIER-LLOBÉRAS, C.; HUMBERT, J. F; COUTÉ, A.; BERNARD, C. Environmental context of Cylindrospermopsis raciborskii (Cyanobacteria) blooms in a shallow pond in France. Water Research, v. 36, n.13, p.3183-3192, 2002.
BRIAND, J. F.; LEBOULANGER, C.; HUMBERT, J. F.; BERNARD, C.; DUFOUR, P. Cylindrospermopsis raciborskii (Cyanobacteria) invasion at midlatitudes: selection, wide physiological tolerance, or global warming? Journal of Phycology, v.40, n.2, p.231-238, 2004.

BUFORD, M. A.; DAVIS, T. W. Physical and chemical processes promoting dominance of the toxic cyanobacterium Cylindrospermopsis raciborskii. Chinese Journal of Oceanology and Limnology, v.29, n.4, p.883-891, 2011.

BURFORD, M. A.; MCNEALE, K. L.; MCKENZIE-SMITH, F. J. The role of nitrogen in promoting the toxisc cyanophyte Cylindrospermopsis raciborskii in a subtropical water reservoir. Freshwater Biology, v.51, n.11, p.2143-2153, 2006.

CARLSON, R. E. A trophic state index for lakes. Limnology and Oceanography, v.22, n.2, p.361-369. 1977.

CHELLAPPA, N. T.; CHELLAPPA, T.; CÂMARA, F. R. A.; ROCHA, O.; CHELLAPPA, S. Impact of stress and disturbance factores on the phytoplankton communities in Northeastern Brazil reservoir. Limnologica, v.39, n.4, p.273282, 2009.

COSTA, D. F.; BARbOSA, J. E. L.; DANTAS, Ê. W. Productivity-diversity relations in phytoplankton reservoir communities in semi-arid region of Northeast Brazil. Journal of Arid Environments, v.126, p.64-70, 2016.

COSTA I. A. S.; CUNHA, S. R. S.; PANOSSO, R.; ARAÚJO, M. F. F.; MELO, J. L. S.; ESKINAZISANT'ANNA, E. M. Dinâmica de Cianobactérias em Reservatórios Eutróficos do Semi-Árido do Rio Grande do Norte. Oecologia Brasiliensis, v.13, n.2, p.382-401, 2009.

DANTAS, Ê. W.; MOURA, A. N.; BITTENCOURTOLIVEIRA, M. C. Cyanobacterial blooms in stratified and destratified eutrophic reservoirs in semi-arid region of Brazil. Anais da Academia Brasileira de Ciências, v.83, n.4, p.13271338, 2011.

FONSECA, B. M. Diversidade fitoplanctônica como discriminador ambiental em dois reservatórios rasos com diferentes estados tróficos no Parque Estadual das Fontes do Ipiranga. 2005. 208f. Tese (Doutorado em Ecologia de Ecossistemas Terrestres e Aquáticos) Universidade de São Paulo, São Paulo. 2005.

GENTIL, C. R. Estrutura da Comunidade fitoplanctônica de pesqueiros da região metropolitana de São Paulo, SP, em dois períodos: primavera e verão. 2007. 134f. Tese de Doutorado (Doutorado em Biodiversidade Vegetal e Meio Ambiente), Faculdade de Saúde Pública da Universidade de São Paulo, São Paulo. 2007.

GENTIL, R. C.; TUCCI, A.; SANT' ANNA, C. L. Dinâmica da comunidade fitoplanctônica e aspectos sanitários de um lago urbano eutrófico em São Paulo, SP. Hoehnea, v.35, n 2, p.265-280, 2008.

HILLEBRAND, H.; DÜRSEKEN, D.; KIRSCHIEL, D.; POLLINGHER, U.; ZOHARY, T. Biovolume calculation for 
pelagic and benthic microalgae. Journal of Phycology, v.35, n.2, p.403-424, 1999.

JEPPESEN, E.; BRUCET, S.; NASELLI-FLORES, L.; PAPASTERGIADOU, E.; STEFANIDIS, K.; NOGES, T.; NOGES, P.; ATTAYDE, J.L.; ZOHARY, T.; COPPENS, J.; BUCAK, T.; MENEZES, R. F.; FREITAS, F. R. S.; KERNAN, M.; SØNDERGAARD, M.; BEKLIOGLU, M. Ecological impacts of global warming and water abstraction on lakes and reservoirs due to changes in water level and related changes in salinity. Hydrobiologia, v.750, n.1, p.201227, 2015.

JESPERSEN, A. M.; CHRISTOFFERSEN, K. Measurements of chlorophyll a from phytoplankton using ethanol as an extraction solvent. Archiv fur Hydrobiologie Jounal, v. 109, p.445-454, 1987.

KOMÁREK, J.; KOMÁRKOVÁ-LEGNEROVÁ, J. Contribuion to the knowledge of planktic cyanoprokaryotes from central México. Preslia, v.74, p.207-233, 2002.

KOMÁREK, J.; ANAGNOSTIDIS, K.; Cyanoprokaryota 2: Oscillatoriales. In: Süsswasserflora Von Mitteleuropa. Elsevier, Spektrum Akademischer Verlag, 2005.

KRUK, C.; MAZZEO, N.; LACEROT, G.; REYNOLDS, C. S. Classification schemes for phytoplankton: A local validation of a functional approach to the analysis of species temporal replacement. Journal of Plankton Research, v.24, n.9, p.901-912, 2002.

LEITÃO A. C.; FREIRE, R. H. F.; ROCHA, O.; SANTAELLA, S. T. Zooplankton community composition and abundance of two Brazilian semiarid reservoir. Acta Limnologica Brasiliensia, v.8, n.4, p.451-468, 2006.

LACHI, G. B.; SIPAÚBA-TAVARES, L. H. Qualidade da água fitoplanctônica de um viveiro de piscicultura utilizado para fins de pesca esportiva e irrigação. Boletim do Instituto de Pesca, v.34, n.1, p.29-38, 2008.

MARINHO, M. M.; HUSZAR, V. L. M. Nutrient availability and physical conditions as controlling factors of phytoplankton composition and biomass in a tropical reservoir (Southeastern Brazil). Archiv für Hydrobiologie, v.15, n.3, p.443-468, 2002.

MEDEIROS, L. D. C.; MATTOS, A.; LÜRLING, M.; BECKER, V. Is the future blue-green or brown? The effects of extreme events on phytoplankton dynamics in a semi-arid man-made lake. Aquatic Ecology, v.49, n.3, p.293-307, 2015.

NASELLI-FLORES， L.; BARONE， R; CHORUS， I.; KURMAYER, R. Toxic cyanobacterial blooms in reservoirs under asemiarid Mediterranean climate: the magnification of a problem. Environmental Toxicology, v.22, n.4, p.399-404, 2007.

O'NEIL, J. M.; DAVIS, T. W.; BURFORD, M. A.; GOBLER, C. J. The rise of harmful cyanobacteria blooms: the potencial roles of eutrophication and climate change. Harmful Algae, v.14, p.313-334, 2012.
PADISÁK, J.; CROSSETTI, L. O.; NASELLI-FLORES, L. Use and misuse in the application of the phytoplankton functional classification: a critical review with updates. Hydrobiologia, v.621, n.1, p.1-19, 2009.

PELECHATA, A.; PELECHATY, M.; PUKACZ, A. Factors influencing cyanobacteria community structure in Charalakes. Ecological Indicators, v.71, p.477-490, 2016.

REYNOLDS, C. S. Vegetation processes in the pelagic: a model for ecosystem theory. Oldendorf: Ecology Institute, 1997.

REYNOLDS, C. S.; HUSZAR, V.; KRUK, C.; NASELLIFLORES, L.; MELO, S. Towards a functional classification of the freshwater phytoplankton. Jounal of Plankton Research, v.24, n.5, p.417-428, 2002.

ROMO, S.; SORIA, J.; FERNÁNDEZ, F.; OUAHID, Y.; BARÓN-SOLÁ, Á. Waters residence time and the dynamic of toxic cyanobacteria. Freshwater Biology, v.58, n.3, p.513$522,2013$.

ROSS, J. Práticas de ecologia. Barcelona: Editorial Omega. 1979

SALMASO, N.; NASELLI-FLORES, L.; PADISÁK, J. Functional classifications and their application in phytoplankton ecology. Freshwater Biology, v.60, n.4, p.603619, 2014.

SANT'ANNA, C. L.; AZEVEDO, M. T. P.; WERNER, W. R.; DOGO, C. R.; RIOS, F. R.; CARVALHO, L. R. Review of toxic specie of Cyanobacteria in Brazil. Algological Studies, v.126, n.1, p.251-265, 2008.

SILVA, R. M. A. Entre o combate à seca e a convivência com o semiárido: políticas públicas e transição paradigmática. Revista Econômica do Nordeste, v.38, n.3, p. 466-485, 2007.

SOARES, M. C. S.; HUSZAR, MIRANDA, M. N.; MELO, M. M.; ROLAND, F.; LURLING, M. Cyanobacterial dominance in Brazil: distribution and environmental preferences. Hydrobiologia v, 717, p. 1-12, 2013.

TOLEDO JÚNIOR, A. P.; TALARICO M.; CHINEZ, S. J.; AGUDO, E. G. A aplicação de modelos simplificados para a avaliação de processo da eutrofização em lagos e reservatórios Tropicais. In: Anais do $12^{\circ}$ Congresso Brasileiro de Engenharia Sanitária. p.1-34,1983.

UTERMÖHL, H. Zur Vervollkommunng der quantitativen phytoplankton methodik. Mitteilungen der Internationale Vereinigung für Theoretische und Angewandte Limnologie, v.9, n.1, p.1-38, 1958.

WETZEL, R. G.; LIKENS, G. E. Limnological Analyses. WB Saunders Co., Philadelphia. 2000. 